\title{
Study of block copolymer micelles as vehicles for hydrophobic drug Lamotrigine
}

\author{
Shilpa Praveen Chaudhari, Jayashree Ramesh Patil. \\ Department of Pharmaceutics,Marathwada Mitra Mandal's College of Pharmacy, Kalewadi, Pune. INDIA
}

\begin{abstract}
Objective: The objective of the study was to investigate the solubilization of poorly water-soluble drug Lamotrigine in pure \& mixed Pluronic polymeric micelles. Method: Two different Pluronic (Pluronic F68, Pluronic L81) were chosen and micelle formulations were prepared by using various drug:polymer ratios and model drug Lamotrigine. Formulations were characterized by critical micellization concentration (CMC) values, cloud point of copolymers, micelle size and size distribution, zeta potential, loading efficiency, drug release and stability. Result: Mixed micelles(hydrophilic and hydrophobic) also helped to overcome the limitations of monosystem of Pluronic L81 and Pluronic F68. The solubilized drug and salt decreased the cloud point of copolymers. Results show that the solubilization of Lamotrigine enhances with the rise in concentration of block copolymers, negative GsO and temperature, but no significant increase was observed with added salt and at a lower $\mathrm{pH}$ the drug show highest solubility. Conclusion: Mixed micelles showed fairly high entrapment efficiency, loading capacity and sustained release profile for Lamotrigine, a model hydrophobe than that of plain Pluronic micelles.
\end{abstract}

Key words: Pluronic F68, Pluronic L81, Micelles, Solubilization.

\section{INTRODUCTION}

In the pharmaceutical technology, attracted incredible attention for development of efficient drug delivery systems during the last two decades. The principal reason for the implausible growth of drug delivery technology is the best prospect for achieving large improvements over current therapies will occur through improved delivery of existing drugs. This necessity arises because of a drug molecule must overcome the enormous barriers before it reaches its target site within the body, where it can perform its biological role. ${ }^{1}$

There are some serious problems associated with the therapeutic application of hydrophobic, poorly water-soluble agents, since low water solubility results in poor absorption and low bioavailability. ${ }^{2}$ In addition, drug aggregation upon intravenous administration of poorly soluble drugs may lead to such complications as embolism ${ }^{3}$ and local toxicity. ${ }^{4}$ To overcome systemic toxicity a very promising approach is the application of drug-loaded nanosized drug carriers, such as micelles, polymeric nanoparticles, dendrimers and liposomes. ${ }^{4,5}$

Polymeric micelles are nano sized assemblies of amphiphilic block copolymers exhibiting an inimitable core-corona structure .The outer hydrophilic shell is important to stabilize the micelles in an aqueous environment and after systemic administration minimize clearance by the mononuclear phagocytic system (MPS) whereas the inner hydrophobic core functions as a drug reservoir. ${ }^{6}$ The assembled polymers are in dynamic equilibrium with free unimers, in contrast to polymeric nanoparticles and the particles are usually smaller $(10-100 \mathrm{~nm})$ displaying monodisperse size distributions ?

Block copolymers are commercially available as linear triblocks of the type ABA and $\mathrm{BAB}$ (Pluronics ${ }^{\circledR}$ ) made of polyethylene oxide (PEO) and polypropylene oxide
Submission Date : 12-09-13 Revision Date : :20-01-14 Accepted Date : :06-11-14

DOI: $10.5530 /$ ijper.48.4s.8 Correspondence Address Prof. Shilpa Praveen

Chaudhari

Marathawada Mitra Mandal's College of Pharmacy, Pune33, INDIA.

Email: shilpapchaudhari78@ yahoo.com

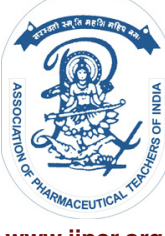

www.ijper.org 
(PPO) with varying hydrophilic-lipophilic balance (HLB).These self-assemble polymeric amphiphiles to form nanosize aggregates (micelles) in aqueous solutions and exhibit a unique core-shell structure which having a fairly low polydispersity that is strongly dependent on temperature/concentration with spherical/rodlike morphology. ${ }^{8,9}$ Pluronic ${ }^{\circledR}$ aggregates have a micelle core consisting of a hydrophobic poly(propylene oxide) or PPO block surrounded by a heavily hydrated, hydrophilic poly(ethylene oxide) or PEO block. ${ }^{9}$ The Pluronics micelles have a slower rate of dissociation and allow retention of loaded drugs for a longer period than other conventional surfactant based drug delivery systems; hence, these surfactants may allow a higher accumulation of the active species at the target site and so Pluronics ${ }^{\circledR}$ have found significant applications in drug delivery systems. ${ }^{10,11}$ The size,morphology and solubilizing capacity of micelles depends on different factors, such as chemical structure of the amphiphile and the drug molecule, $\mathrm{pH}$, temperature, and the ionic strength. ${ }^{12}$ The solubilizing power of polymeric surfactants are generally considered to be better than conventional surfactants due to their low critical micelle concentrations $(\mathrm{CMC})$ and the thermal stability of the micelles. ${ }^{13}$

A variety of block copolymers may be used to form polymeric micelles. ${ }^{14}$ In this study we used block copolymer such as Pluronic F68 and Pluronic L81 are used which are difunctional block copolymer surfactant terminating in primary hydroxyl groups from BASF.

Epilepsy is a common chronic neurological disorder characterized by recurrent unprovoked seizures. ${ }^{15}$ In order to avoid the risk of permanent brain damage which requires quick management of seizures. Parenteral administration allows transport of drugs to the brain abstaining BBB, thus providing a unique feature and better option to target drugs (for example Lamotrigine) to the brain with rapid onset of action in case of emergencies such as epilepsy.

Lamotrigine is an anticonvulsant drug used in the treatment of epilepsy and bipolar disorder and widely used as mono or adjunct therapy in adults and children. The Lamotrigine a (biopharmaceutical classification system) BCS class II drug is currently available as a tablet which is administered 2-3 times per day as divided doses of $25-600 \mathrm{mg}^{16}$

Mixed micelles reveal synergistic properties, such as increased micelle stability and drug loading efficiency, superior to those of the individual components. ${ }^{17}$ In this study, mixed micelles made of hydrophobic Pluronic L81 and hydrophilic Pluronic F68 and pure micelles of individual component with Lamotrigine were prepared and also studied the solubilization efficiency of
Lamotrigine for binary mixture of PluronicsF68 and Pluronic L81. The mixed micelles of triblock copolymer surfactant Pluronic F68, Pluronic L81 with Lamotrigine has been studied as a function of temperature, presence of electrolyte $(\mathrm{NaCl})$ and different $\mathrm{pH}$

\section{MATERIAL AND METHOD}

\section{Materials}

Pluronics (Pluronics f68 and Pluronic L81) were purchased from Sigma-Aldrich.Lamotrigine was gift sample from Kopran Pharmaceuticals,Mumbai.All other chemicals and components for buffer solutions were of analytical grade.

\section{Study of Block Polymers for Solubilization (CMC)}

Surface tension method was used for CMC determination of Pluronic F68 and Pluronic L81 polymers. The $\mathrm{CMC}$ of the block copolymer at $25^{\circ}$ was determined in pure water.The $\mathrm{CMC}$ determinations for Pluronic F68, Pluronic L81were based on the change in surface tension with surfactant concentration. Stalagnometer was used to measure the surface tension. Each surface tension measurement was repeated three times, and the typical error in the $\mathrm{CMC}$ determination was less than $5 \%$.

\section{Cloud point (CP)}

Cloud points of optimized formulation with and without drug were determined in the presence and absence of varying amounts of added sodium chloride by gradually heating the solution in thin $20 \mathrm{~mL}$ glass tubes immersed in a temperature controlled water bath. The solutions were stirred with a magnetic stirring bar while being heated. The heating rate of the samples was adjusted to $1{ }^{\circ} \mathrm{C} / \mathrm{min}$. The first appearance of turbidity was taken as the cloud point and cloud point values were found to be reproducible within $0.5^{\circ} \mathrm{C}$.

\section{Preparation of drug-loaded polymeric micelles}

Polymeric micelles containing Lamotrigine were prepared by direct dissolution technique using block copolymer (Pluronic L81, Pluronic F68) alone and in combination (1:1) ratio. Block copolymer was dissolved in water, Lamotrigine was then added to the polymer solution. The solution was stirred continuously on stirrer at room temperature at different rpm for period of $6 \mathrm{~h}$ to optimize the RPM for preparation of micelles. Nonincorporated drug remained insoluble and was separated by filteration of micelle suspension through a $0.2 \mu \mathrm{m}$ filter. The filtered solution was diluted 10 times with polymeric solution. The Lamotrigine concentration in filtered solution was estimated by measuring UV absorbance at $\lambda \max 306 \mathrm{~nm}^{17}$ 


\section{Table 1: Factors and Levels for the $\mathbf{3 3}$ factorial design}

\begin{tabular}{|c|c|c|c|c|}
\hline Independent Variables & Parameters & -1 & $\mathbf{0}$ & $\mathbf{+ 1}$ \\
\cline { 3 - 5 } & & $1: 1$ & $1: 5$ & $1: 10$ \\
\hline X1 & Polymer:Drug ratio & 12 & 24 & 48 \\
\hline X2 & Time of stirring & Pluronic F68 & Pluronic L81 & Pluronic F68:L81(1:1) \\
\hline X3 & Type of block copolymer & \multicolumn{3}{|c|}{ Dependent Variables } \\
\hline Y1 & \multicolumn{3}{|c}{} \\
\hline
\end{tabular}

\begin{tabular}{|c|c|c|c|}
\hline Batch code & \multicolumn{3}{|c|}{ Variables levels in coaded form } \\
\hline $\mathbf{F}$ & $\mathrm{X} 1$ & $\mathrm{x} 2$ & $\mathrm{X} 3$ \\
\hline F1 & 1 & 1 & 1 \\
\hline F2 & -1 & 0 & 1 \\
\hline F3 & 0 & 0 & -1 \\
\hline F4 & -1 & -1 & 1 \\
\hline F5 & 1 & 0 & -1 \\
\hline F6 & -1 & 1 & 0 \\
\hline F7 & 1 & -1 & 1 \\
\hline F8 & 1 & -1 & -1 \\
\hline F9 & 0 & 0 & 0 \\
\hline F10 & -1 & 0 & -1 \\
\hline F11 & -1 & -1 & 0 \\
\hline F12 & -1 & 0 & 0 \\
\hline F13 & 0 & 1 & 0 \\
\hline F14 & 1 & -1 & 0 \\
\hline F15 & 0 & 1 & -1 \\
\hline F16 & 0 & -1 & 0 \\
\hline F17 & 1 & 1 & 0 \\
\hline F18 & 1 & 1 & -1 \\
\hline F19 & -1 & 1 & 1 \\
\hline F20 & 0 & -1 & -1 \\
\hline F21 & 1 & 0 & 1 , \\
\hline F22 & 1 & 0 & 0 \\
\hline F23 & 0 & 0 & 1 \\
\hline F24 & -1 & 1 & -1 \\
\hline F25 & -1 & -1 & -1 \\
\hline F26 & 0 & -1 & 0 \\
\hline F27 & 0 & 1 & 0 \\
\hline
\end{tabular}

\section{Optimization of polymeric micelles}

Twenty seven Lamotrigine polymeric micelles formulation were prepared according to $3^{3}$ factorial design employing the factors and levels shown in (Table1and2).

\section{Study the effect of operating variables on polymeric micelles}

Polymeric micelles were prepared at different temperature $\left(30,35,45^{\circ} \mathrm{C}\right), \mathrm{pH}(2,6,8)$, and salt $(\mathrm{NaCl})$ concen- trations $(1.45 \%, 0.9 \%, 0.45 \%)$ keeping the stirring time constant i.e., 6 h. The effect of temperature, $\mathrm{pH}$, and salt on the percentage of drug entrapment in polymeric micelles was calculated.

\section{Drug entrapment}

The filtered solution of drug containing polymeric micelles was diluted 10 times with methanol. Blank experiments, without Lamotrigine, were done to determine the solubility of the drug in water.The Lamotrig- 
ine concentration in polymeric solution was estimated by measuring UV absorbance at $\lambda \max 306 \mathrm{~nm}$.

After estimating the drug contents by UV, incorporated drug (\%) and drug weight (\%) in micelles were calculated using Equations (1) and (2):

Drug incorporated $(\%)=(a / b) * 100$

Drug weight in micelle $=(\mathrm{a} /(\mathrm{b}+\mathrm{c})) * 100$

where $\mathrm{a}$ is the amount of drug loaded in micelle $(\mathrm{g}), \mathrm{b}$ the amount of drug used in micelle preparation $(\mathrm{g})$ and $c$ the amount of polymer used in micelle preparation (g).

\section{Determination of micelles size and polydispersity index}

The micelles size determination as performed using photon correlation spectroscopy within built zetasizer (model: nano zs, malvern instrument, USA). Aliquot preconcentrate $(0.5 \mathrm{~mL})$ was diluted to $50 \mathrm{~mL}$ with distill water; stir slowly to form a dispersion. Diluted samples were directly placed in to the module for measurements. All determination is made in triplicate.

\section{Zeta potential determination}

Sample $(0.5 \mathrm{~mL})$ was diluted to $50 \mathrm{~mL}$ with distill water in glass beaker with constant stirring. Zeta potential of the resultant solution was determined using the zetasizer (model: nano zs, malvern instrument, USA ). All determination is made in triplicate.

In vitro release of lamotrigine from polymeric micelles across the cellophane membrane

The release of Lamotrigine from polymeric micelles was determined using the membrane diffusion technique, 1 $\mathrm{ml}$ of polymeric micelles suspension was placed in a diffusion cell (glass tube) of diameter $2.5 \mathrm{~cm}$, the lower open end of the glass tube was covered with soaked cellulose membrane. This cell then filled with phosphate buffer solution $\mathrm{pH} 6$ and $8(25 \mathrm{ml})$. This was constantly stirred at speed $50 \mathrm{rpm}$ at $37 \pm 10^{\circ}$ on a magnetic stirrer with a thermostat. Aliquots were withdrawn at 15, 30, $60,90,120, \mathrm{~min}$ (up to 6 hours) intervals and replaced simultaneously with equal volume of fresh phosphate buffer solution. The Lamotrigine concentration in the samples was analyzed spectrophotometrically, at wavelength $306 \mathrm{~nm}$ to determine the amount of drug release.

\section{Stability studies}

Stability test of micelles consisted of visual control and analytical measurement of drug content. For this purpose, lyophilized and aqueous solution forms of Lamotrigine loaded micelles were placed in a stability chamber at $25 \pm 2{ }^{\circ}, 60 \%$ relative humidity for 3 months. Lyophilized forms were placed in $2 \mathrm{ml}$ Eppendorf tubes and solution forms were placed in $5 \mathrm{ml}$ bottle with a lid. Samples were taken to determine the drug content at the beginning and at the end of 3 months. Drug content was measured spectrophotometrically after extracting drug into methanol and UV spectrums were also checked. $^{10}$

\section{RESULT AND DISCUSSION}

\section{Determination of critical micelle concentration}

The critical micelle concentration can be determined by carrying out surface tension measurement using stalagnometer by drop count method on a series of different Pluronic concentration, such as for F68 $1 \mathrm{mg} / \mathrm{ml}$ to $8 \mathrm{mg} / \mathrm{ml}$ and for L81 from $10 \mu \mathrm{g} / \mathrm{ml}$ to $80 \mu \mathrm{g} / \mathrm{ml}$. Surfactant exhibit a specific surface tension curve as a function of the concentration. Initially the surfactant molecules increasingly enrich themselves at the water surface. During this phase the surface tension decreases from $59.79 \mathrm{~N} / \mathrm{m}$ to $50.15 \mathrm{~N} / \mathrm{m}$ for F68 and $41.28 \mathrm{~N} / \mathrm{m}$ to $37.65 \mathrm{~N} / \mathrm{m}$ for L81 linearly with the logarithm of the surfactant concentration. when the critical micelle concentration is reached, a further increase in surfactant concentration no longer has any considerable influence on the surface tension. The obtained critical micelle concentration values of polymers in aqueous solution were $4 \mathrm{mg} / \mathrm{ml}$ and $0.06 \mathrm{mg} / \mathrm{ml}$ for Pluronic F68 and Pluronic L81.

\begin{tabular}{|c|c|c|c|c|c|c|c|c|}
\hline \multirow{3}{*}{$\begin{array}{l}\text { Type of Block } \\
\text { polymer }\end{array}$} & \multicolumn{8}{|c|}{ Cloud point $\left({ }^{\circ} \mathrm{C}\right)$} \\
\hline & \multicolumn{2}{|c|}{ Distilled Water } & \multicolumn{2}{|c|}{$\begin{array}{l}0.45 \% \text { w/v } \mathrm{NaCl} \\
\text { solution }\end{array}$} & \multicolumn{2}{|c|}{$0.9 \% w / v ~ N a C l$ solution } & \multicolumn{2}{|c|}{$1.45 \% \mathrm{w} / \mathrm{v} \mathrm{NaCl}$ solution } \\
\hline & $\begin{array}{c}\text { Without } \\
\text { drug }\end{array}$ & $\begin{array}{c}\text { Drug } \\
\text { saturated }\end{array}$ & $\begin{array}{c}\text { Without } \\
\text { drug }\end{array}$ & $\begin{array}{l}\text { Drug } \\
\text { saturated }\end{array}$ & $\begin{array}{c}\text { Without } \\
\text { drug }\end{array}$ & $\begin{array}{c}\text { Drug } \\
\text { saturated }\end{array}$ & $\begin{array}{c}\text { Without } \\
\text { drug }\end{array}$ & $\begin{array}{c}\text { Drug } \\
\text { saturated }\end{array}$ \\
\hline Pluronic L81(1.25\%) & 21 & 20 & 20 & 18 & 19 & 18 & 18 & 17 \\
\hline Pluronic F68 (2.5\%) & \multicolumn{8}{|c|}{$100<$} \\
\hline $\begin{array}{c}\text { Combination } \\
\text { of Pluronic } \\
\text { L81,F68(1:1) }(2.25 \%)\end{array}$ & 23 & 21 & 22 & 21 & 21 & 20 & 20 & 18 \\
\hline
\end{tabular}




\section{Cloud point}

The cloud point of nonionic surfactant depend on its molecular structures i.e, the lipophilic nonpolar and the hydrophilic parts (PEO). Increasing hydrophobicity decreases the cloud point whereas increasing the hydrophilicity increases the cloud point. ${ }^{18}$ The data presented in (Table 3) for cloud point (CP) of $1.25 \%$ of pure Pluronic L81, 2.25\% of mixed Pluronic L81and Pluronic F68 with and without drug (Lamotrigine) in water and salt solutions with this behavior. An almost linear decrease in the cloud point of two copolymers and their combination with increasing salt concentration and. The effect was even more when copolymers are saturated with the drug (Lamotrigine) for same salt concentration. The solutions remain simply miceller without any anisotrophy below the cloud point but transform into two phase system above it. At temperature below about $20^{\circ}$, even micelles are absent and the copolymer remains dissolved. At the molecular level because of the hydrophilic nature of PPO in water at lower temperature. The micelle formation of EO-PO block copolymers is mainly dependent on concentration, temperature and hydrophobic interactions. Micelles grew large, particular at temperature near the cloud point. Salt are known to change the cloud point of water soluble polymers such as PEO, PPO based nonionic surfactant. The effect has been attributed due to their salting out action by dehydrating the PEO shell, similar to the effect of temperature and increases the hydrophobicity in the PPO block of the copolymer. ${ }^{18}$

\section{Preparation of polymeric micelles}

Optimization of RPM on the basis of highest drug loading for 6hrs was done ,since the optimized RPM for plain pluronic L81and combination of (pluronic L81, pluronic F68) was 600 RPM and 900 RPM for plain pluronic F68 micelles respectively, hence these were selected for the further study.

\section{Loading of drugs in polymeric micelles}

$\%$ drug loading was performed using $3^{3}$ factorial design for which the results are as shown in (Table 4) and (Figure 1 and 2)

Statistical analysis: The polynomial equation for 2F1model as given below:

$Y=A_{0}+A_{1} X_{1}+A_{2} X_{2}+A_{3} X_{3}+A_{12} X_{1} X_{2}+A_{13} X_{1} X_{3}+A_{23} X_{2} X_{3}$ $+\mathrm{A}_{11} \mathrm{X}_{1}^{2}+\mathrm{A}_{22} \mathrm{X}_{2}^{2}+\mathrm{A}_{33} \mathrm{X}_{3}^{2}$

\begin{tabular}{|c|c|c|c|c|c|c|}
\hline \multicolumn{7}{|c|}{ Table 4: Summary of result of regression analysis for response Y. } \\
\hline Model & R2 & Adjusted R2 & Predicted R2 & S.D. & Remarks \\
\hline 2F1 & 0.9838 & 0.9472 & 0.8150 & 1.72 & Suggested \\
\hline
\end{tabular}
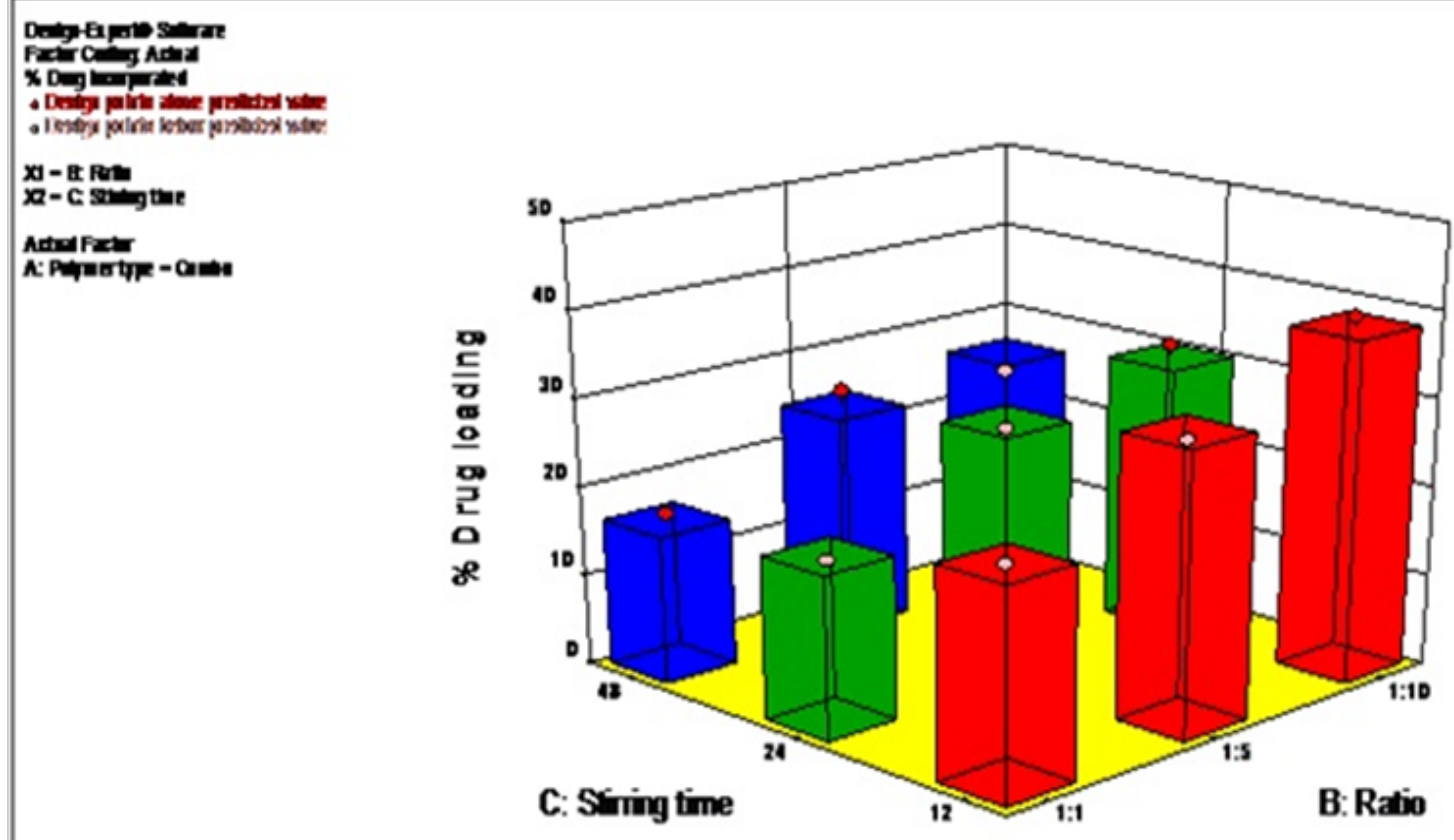

Figure 1: 3D graph of factor X1 and X2 for \% drug loading 


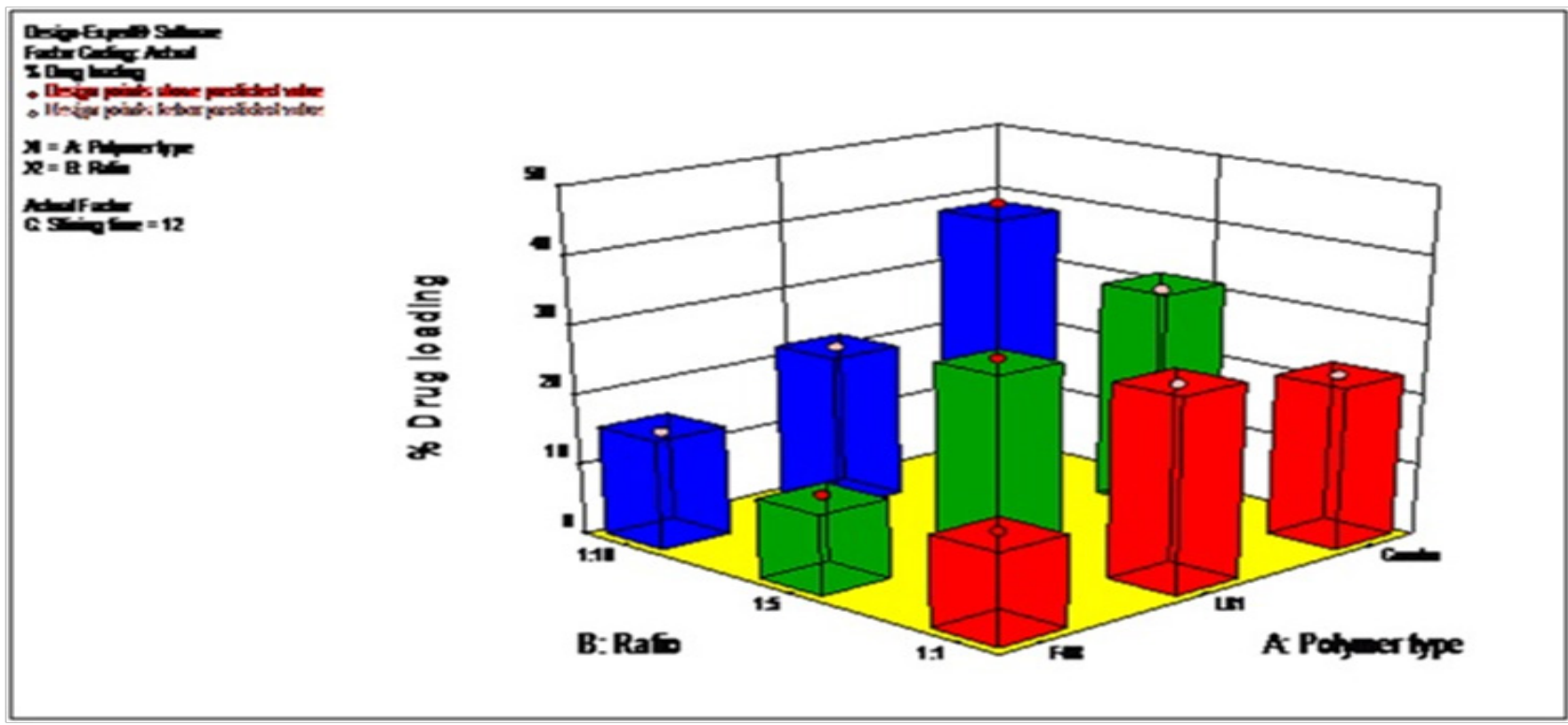

Figure 2: 3D graph of factor $X 1$ and $X 3$ for $\%$ drug loading

$\mathrm{Y}=23.80+(-6.26) \mathrm{X} 1 \mathrm{a}+3.34 \mathrm{X} 1 \mathrm{~b}+(-1.15) \mathrm{X} 2 \mathrm{a}+0.69 \mathrm{X} 2 \mathrm{~b}+(-0.39) \mathrm{X} 3 \mathrm{a}+(-2.39) \mathrm{X} 3 \mathrm{~b}+1$ $.46 \times 1 \mathrm{a} \times 2 \mathrm{a}+4.47 \times 1 \mathrm{~b} 2 \mathrm{a}+(-1.54) \times 1 \mathrm{a} \times 2 \mathrm{~b}+0.55 \times 1 \mathrm{~b} 2 \mathrm{~b}+(-3.67) \times 1 \mathrm{a} \times 3 \mathrm{a}+(-1.45)$ $\mathrm{X} 1 \mathrm{bX} 3 \mathrm{a}+1.24 \mathrm{X} 1 \mathrm{aX} 3 \mathrm{~b}+(-2.92) \times 1 \mathrm{bX} 3 \mathrm{~b}+(-0.82) \times 2 \mathrm{aX} 3 \mathrm{a}+(-1.12) \times 2 \mathrm{~b} 3 \mathrm{a}+(-0.24)$ $\mathrm{X} 2 \mathrm{aX} 3 \mathrm{~b}+0.64 \mathrm{X} 2 \mathrm{bX} 3 \mathrm{~b}$

Hydrophobic micelle core works as a cargo to improve solubility and stability of lipophilic drugs. Drugs loaded into micelle core by hydrophobic interactions and covalent/non-covalent bond formation. Consequently, depending on both hydrophobic interactions and steric properties it is not possible to load all kind of drugs into polymeric micelles. Several of the major factors which influence the loading capacity and loading efficiency of block copolymer micelles are nature of the solute, nature of the core forming block, core block length, total copolymer molecular weight, solute concentration and to a lesser extent, the nature and block length of the outer micelle shell. ${ }^{14}$ For reasons, we studied these two different polymer and their combinations. In both polymer types, no loading efficiency was obtained. Changing the drug:polymer ratios contribute to loading. Lamotrigine was loaded into micelles by direct dissolution. The maximum loading efficiency of $31.5 \%$ was obtained by loading Lamotrigine into Pluronic L81. Loading percent into Pluronic F68 micelles was less than Pluronic L81 micelles (Figure2). With further increase in polymer:drug ratio from 1:1 to 10:1, there was an increase in loading efficiency of drug with both polymers. This might show that amount of added Lamotrigine was not exceeding the drug-carrying capacity of the core in these micelles. Contrary to Pluronic F68, in Pluronic L81 no regular increase on loading efficiency was observed with increasing polymer ratio. The interpenetrating network formed stabilized the micelles at concentrations below the critical micellar concentration of free polymer, though the increased micellar stability was not permanent and disappeared over a time period of days to weeks. In order to overcome lack of stability we introduced the concept of binary mixing of hydrophobic (L81) and hydrophilic (F68) Pluronic block copolymers. Even though, large aggregates were primarily formed and phase separation apparent during the first 24-48 h, sonication (1-2 min) stabilized the dispersions. A combination of pluronic L81/ F68 (1:1\% weight ratio) formed stable dispersions with a small particle size and displayed higher solubilization capacity of a hydrophobic drug compared to that of pure Pluronic F68 and Pluronic L81 micelles.Pluronic L81 contains 10 $\%$ hydrophilic and $90 \%$ hydrophobic residue ${ }^{19,20}$ aggregated with a lamella structure. The hydrophobic block, PPO of Pluronic L81, aggregated due to its hydrophobic interaction in aqueous solution, forming a supramacromolecular structure. However, the hydrophilic block PEO of Pluronic L81 only has 10\% of the whole molecolar weight, not providing sufficient steric hindrance to form a stable dispersion. In this study,Pluronic F68 (EO76-PO30-EO76), ${ }^{20,21}$ was admixed into Pluronic L81 to prepare a stable micelle. The Pluronic F68 with long chains of PEO kinetically provided high stability and spherical structure for Pluronic L81. However, the stability pattern for formulation to those prepared with and without sonication was similar

\section{Effect of variables on optimized formulation (combination 1:10)}

Lamotrigine solubilized in block copolymer.The amount of drug solubilized was determined at different temper- 


\begin{tabular}{|c|c|c|c|c|c|c|c|c|c|}
\hline \multirow[t]{2}{*}{ Parameters } & \multicolumn{3}{|c|}{ Temperature $\left({ }^{\circ} \mathrm{C}\right)$} & \multicolumn{3}{|c|}{$\mathrm{pH}$} & \multicolumn{3}{|c|}{ Salt concentration (\%) } \\
\hline & 25 & 35 & 45 & 2 & 6 & 10 & 0.46 & 0.9 & 1.46 \\
\hline $\begin{array}{c}\text { Drug } \\
\text { incorporated(\%) } \pm S E^{\mathrm{a}}\end{array}$ & 25.16 & 32.2 & 39.3 & 34.42 & 26.47 & 24.9 & 34.93 & 34.3 & 29.35 \\
\hline $\begin{array}{l}\text { Drug weight in } \\
\text { micelles (\%) }\end{array}$ & 12.58 & 16.1 & 19.75 & 17.21 & 13.23 & 12.45 & 17.46 & 17.25 & 16.675 \\
\hline
\end{tabular}

ature, $\mathrm{pH}$ and salt concentration. Lamotrigine solubilization monitored using UVspectroscopy and the results reveal a strong intereaction between drug and copolymer .The solubility of drug increases with copolymer concentration, temperature and was also influence by changing salt concentration and $\mathrm{pH}$ of the solution and data presented in (Table 5).

\section{Effect of temperature}

With an increased in temperature the amount of drug solubilised in miceller system increases due to miceller growth. The apparent concentration of Lamotrigine in optimized formulation as a function of temperature is shown in fig.(3).The solubility of Lamotrigine in water at $25^{0}$ is $0.17 \mathrm{mg} / \mathrm{ml} .{ }^{22}$ The solubility of Lamotrigine in Pluronic solution significantly increases with an increase in temperature. In general, with an increase in temperature, the dehydration of the PEO shell occurs and PPO chains become more hydrophobic, the drug might be expected to be solubilized in micellar core which is confirmed by UV spectroscopy due to the micellar environment is different than that of water.

\section{Thermodynamic of solubilisation}

Solubilization properties of the polymeric micelles are usually expressed in terms of micelle-water partition coefficient. Thermodynamic parameters are particularly useful in understanding the solubilization of hydrophobic species like Lamotrigine in block copolymer. The ratio of the drug concentration in the micelle to that in water for a particular copolymer concentration, as shown below;

$P=S_{t o t}-S_{w} / S_{w}$

Where $S_{\text {tot }}$ and $S_{w}$ are concentrations of drugs in the micelles and in the water, respectively. The colloidal surfactants usually do not change the chemical poten- tial of the solute, therefore the solubility of Lamotrigine in the extramicellar phase was assumed to be equal to water $(0.0533 \mathrm{mg} / \mathrm{ml})$.Higher the partition coefficient the better hydrophobic microenvironment formed by the combination block co-polymers. The standard Gibbs energy of solubilization $\left(\Delta \mathrm{G}_{\mathrm{s}}{ }^{\mathrm{g}}\right)$ represents the energy change for the transfer of one mole of lamotrigine drug from a hydrophilic environment to the hydrophobic environment is given by

$\Delta G_{s}^{0}=-R T \operatorname{Ln} P$

Where $\mathrm{R}$ is the gas constant, $\mathrm{P}$ is the partition coefficient of the drug between the micelle and aqueous phase and solubilization can be considered as normal partitioning of the drug between the micelle and bulk phases, $T$ is the temperature on the Kelvin scale.The standard Enthalpy and entropy of solubilization process were determined by equations 3 and 4 respectively,as shown below:

$$
\begin{aligned}
& \Delta H_{s}^{0}=-\mathrm{R}[d \mathrm{~L} n \mathrm{P} / d(1 / \mathrm{T})] \\
& \Delta S_{s}^{0}=\Delta H_{s}^{0}-\Delta G_{s}^{0} / T
\end{aligned}
$$

The thermodynamic parameters and micelle-water partition coefficient $(\mathrm{P})$ for solubilization of Lamotrigine in block copolymer solutions as a function of concentration are shown in (Table 6). The values of Gibbs energy of solubilization $\left(\Delta \mathrm{G}_{\mathrm{s}}{ }^{\mathrm{g}}\right)$ are negative, indicating that more and more water molecules leach out from the micelle core and generating a more hydrophobic environment and with increasing block copolymer and the temperature the drug molecule spontaneously partitions into the micelles. The positive value of $\Delta \mathrm{S}_{\mathrm{S}}{ }^{0}$ might be due to liberation of water molecules surrounding the drug when it is partitions from the aqueous phase to micelles. It also indicates that partitioning of the drug is enthalpically disfavoured process. Thus the solubiliza-

\begin{tabular}{|c|c|c|c|c|}
\hline & & luronicL81,Fe & & \\
\hline Temperature, $\mathbf{k}$ & Partition coefficient & $\Delta \mathbf{G S}^{0}, \mathrm{KJ} \mathrm{mol}^{-1}$ & $\Delta \mathrm{HS}^{0}, \mathrm{KJ} \mathrm{mol}^{-1}$ & $\Delta \mathrm{SS}^{0}, \mathrm{~J} \mathrm{~mol}^{-1} \mathbf{k}^{-1}$ \\
\hline 303 & 2.698529 & -2.50091 & \multirow{3}{*}{66.64921} & 0.228218 \\
\hline 308 & 3.735294 & -3.37477 & & 0.227351 \\
\hline 318 & 4.883824 & -4.1932 & & 0.222775 \\
\hline
\end{tabular}
tion was found to be endothermic in nature. 
Table 7: Micelles size,zeta potential and polydispersity index of polymeric micelles

\begin{tabular}{|c|c|c|c|}
\hline Formulation & Micelles size $(\mathbf{n m})$ & Zeta potential $(\mathbf{m v})$ & Polydispersity index \\
\hline F68(1:1) & 221.5 & -25.34 & 0.614 \\
\hline L81(1:5) & 187.9 & -7.35 & 0.557 \\
\hline Combination1:10 & 384.7 & -4.37 & 0.491 \\
\hline Effect of salt0.46\% & 246.5 & -19.45 & 0.576 \\
\hline Effect of salt0.9\% & 346.5 & -18.87 & 0.576 \\
\hline Effect of temp25 & $>1 \mu \mathrm{m}$ & -7.34 & 0.531 \\
\hline Effect of temp 45 & 439.4 & -23.24 & 0.289 \\
\hline Effect of $\mathbf{p H 2}$ & $>1 \mu \mathrm{m}$ & -21.34 & 0.661 \\
\hline Effect of $\mathbf{p H 6}$ & 1082 & -13.34 & 0.465 \\
\hline Effect of $\mathbf{p H 1 0}$ & $>1 \mu \mathrm{m}$ & -34.14 & 0.542 \\
\hline
\end{tabular}

\section{Effect of $\mathrm{pH}$}

The percent entrapment of Lamotrigine increases with the decrease in $\mathrm{pH}$ from 10 to 2 for mixed pluronic micelles. At lower $\mathrm{pH}$ the entrapment of Lamotrigine is significantly increased from $24.9 \%$ at $\mathrm{pH} 10$ to $34.42 \%$ at $\mathrm{pH}$. This can be explained by the fact that Lamotrigine is present as an ionized molecule at lower $\mathrm{pH}$.

\section{Effect of salt}

The percent of the solubilized drug Lamotrigine was decreased in the presence of salt. On the addition of salt the Pluronics become more hydrophobic in nature i.e it contains fewer EO groups than PO groups. Since, the PPO core loses most of the associated water molecules. The PEO core would shrink due to dehydration and fall on the PPO core and it creating problem for the drug to incorporate in to the hydrophobic core of the aggregates. ${ }^{23}$ Hence, we would expect that the partitioning of the drug inside the micelles would not be favoured, and thus, noticeable solubility of Lamotrigine is not observed.

Every proton containing chemical has a unique set of proton NMR signal. These signals may be affected by (eg.temperature, $\mathrm{pH}$,hydrophobicity etc) nature of environment surrounding them transfer of protons from a polar region to a nonpolar region would be charecterized by low frequency shift.

Effect of different concentration on the chemical shifts $(\delta)$ of the $\mathrm{PO}-\mathrm{CH}_{3}$ and $\mathrm{EO}-\mathrm{CH}_{2}$ - signals are presented in fig.5. Addition of salt in different concentration extract water molecule from Pluronic core so at room temperature these protons become dehydrated and exhibit low frequency shifts in the peak,a remarkable decrease in chemical shifts of PO segments display that is greater the addition of salt,the larger the low frequency chemical shift of the block copolymer, which is similar to the behavior observed for the change in EO segments.

\section{Micelles size and polydispersity index}

(Table 7) shows the hydrodynamic size of plain micelles of Pluronic F68,Pluronic L81and their mixed micelles and also depicts the effect of salt, $\mathrm{pH}$ and temperature on hydrodynamic size of optimized mixed micelles system, the size increases on addition of salt, the solubilization capacity was found to decreases. The micelles size as well as polydispersity index decreases on increasing temperature. Whereas with increases or decreases in $\mathrm{pH}$ the micelles size as well as polydispersity index increases.

\section{Zeta potential}

Zeta potential of lamotrigine-loaded micelles was measured as in (Table 7). It was shown that formulations have negative surface charges. Zeta potential of Pluronics and their combination micelles was within the ranges from -4.37 to-34.14 MV.The absolute value of zeta potential decreases. The zeta potential of mixed micelles showed that they are more stable against aggregation than pure Pluronic f68 and Pluronic L81 micelles.

\section{Drug release}

In vitro drug release experiments were carried out on Pluronic L81 and Pluronic F68 micelles and their mixed polymeric micelles. The release profiles of Lamotrigine from pure and mixed Pluronic micelles were evaluated at $\mathrm{pH} 8$ and 6 over 6 hours by a simple diffusion method \& were shown in (Figure.3\&4). The percentages of Lamotrigine released from pure Pluronic F68 and Pluronic L81 Lamotrigine micelles after 6hours at pH 6 and 8 were $92.9 \%, 65.75 \%$ and $80.03 \%, 61 \%$ and their combination were $59.36 \%, 36.47 \%$. The release data showed that Lamotrigine release is statistically significantly higher at $\mathrm{pH} 6$ than $\mathrm{pH} 8$ (Figure.5and6). The Lamotrigine release behavior indicated the Lamotrigine incorporation stability and could be explained through the Lamotrigine location within the micelles. In case of plain Pluronic micelles Lamotrigine release rate more quick than that of mixed micelles. Hence the release 


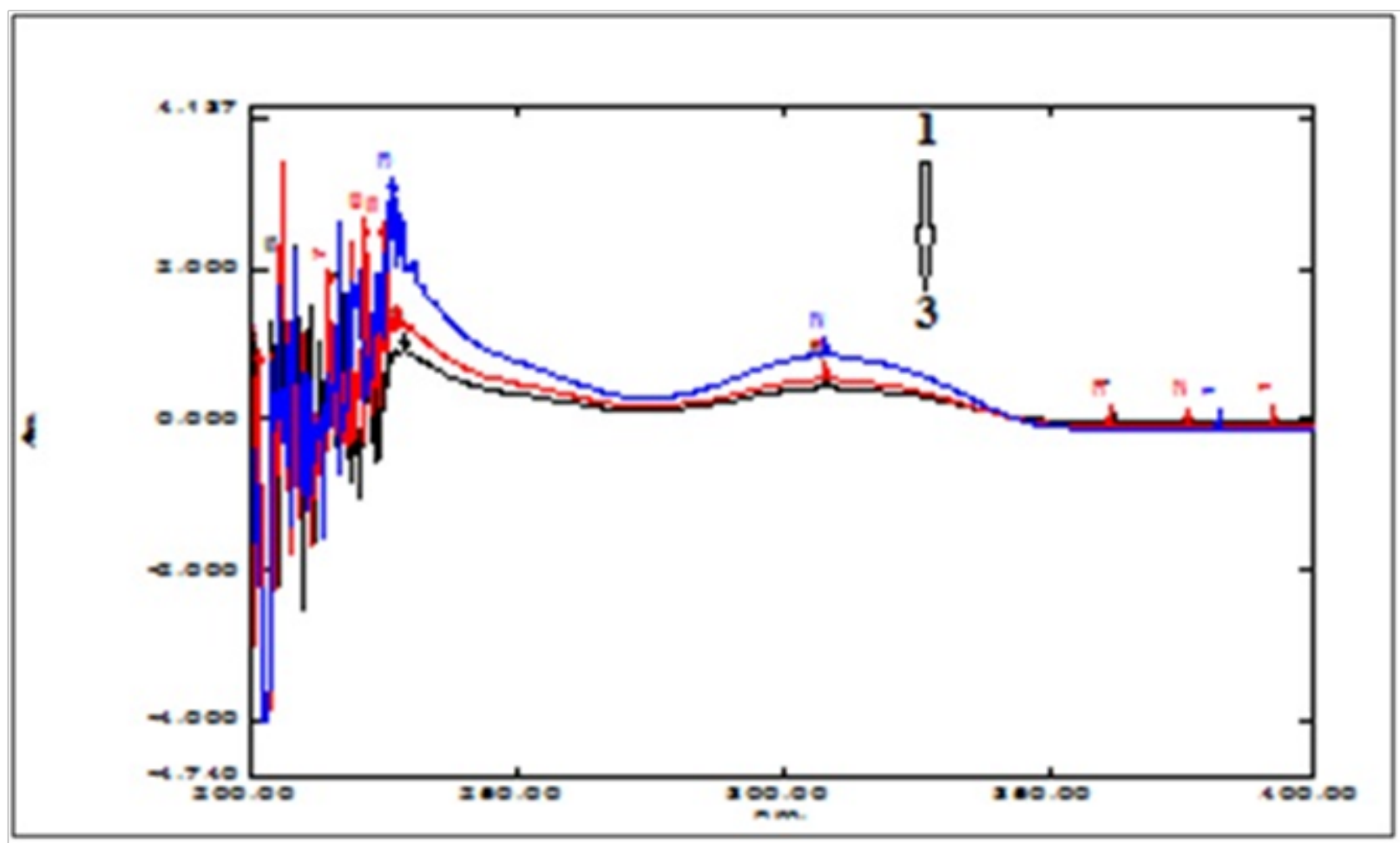

Figure 3: UV vis spectra of solubilized drug Lamotrigine $\left(\lambda_{\max }=306 \mathrm{~nm}\right)$ in mixed micelles at different temperature :1) $\left.45^{\circ} 2\right) 35$ 03) $25^{\circ} \mathrm{C}$

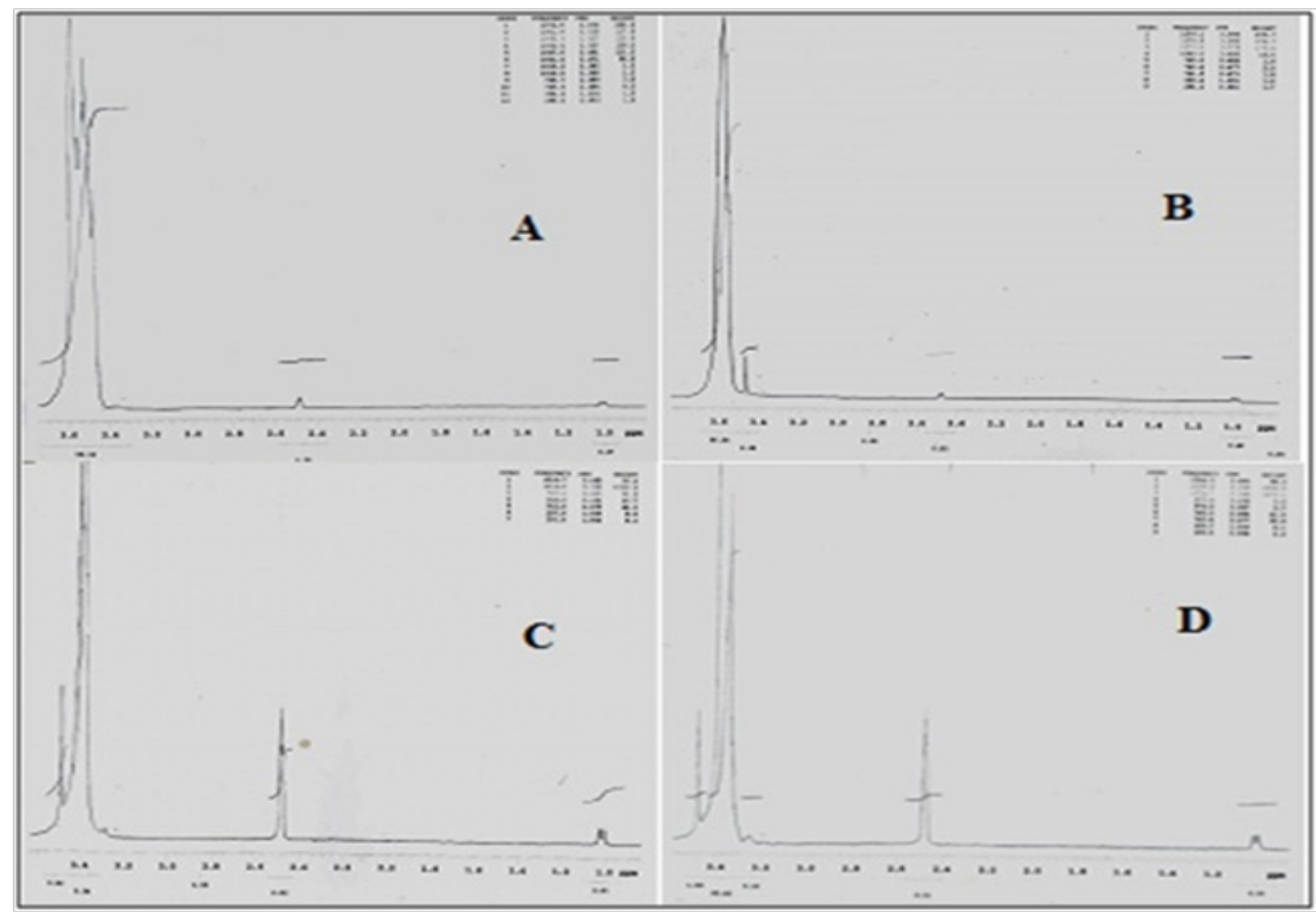

Figure 4: $1 \mathrm{H}$ NMR spectra of mixed pluronic micelles in DMSO solution showing A) $0 \% \mathrm{NaCl} B) 0.46 \%$ C) $0.9 \%$ D) $1.46 \%$ 


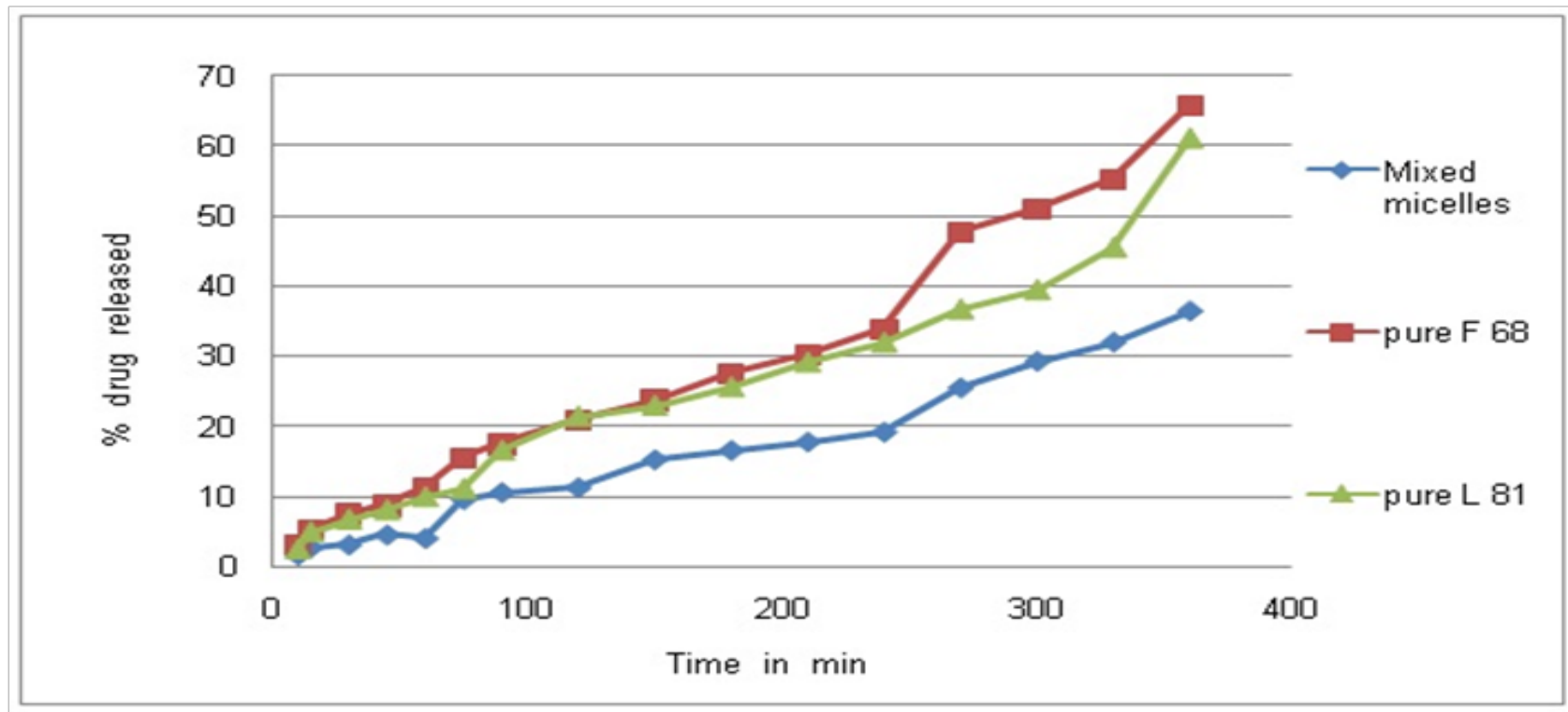

Figure 5: Comparison of drug release profiles of mono Pluronic F 68,Pluronic L81 micelles and their mixed micelles in pH 8 Buffer

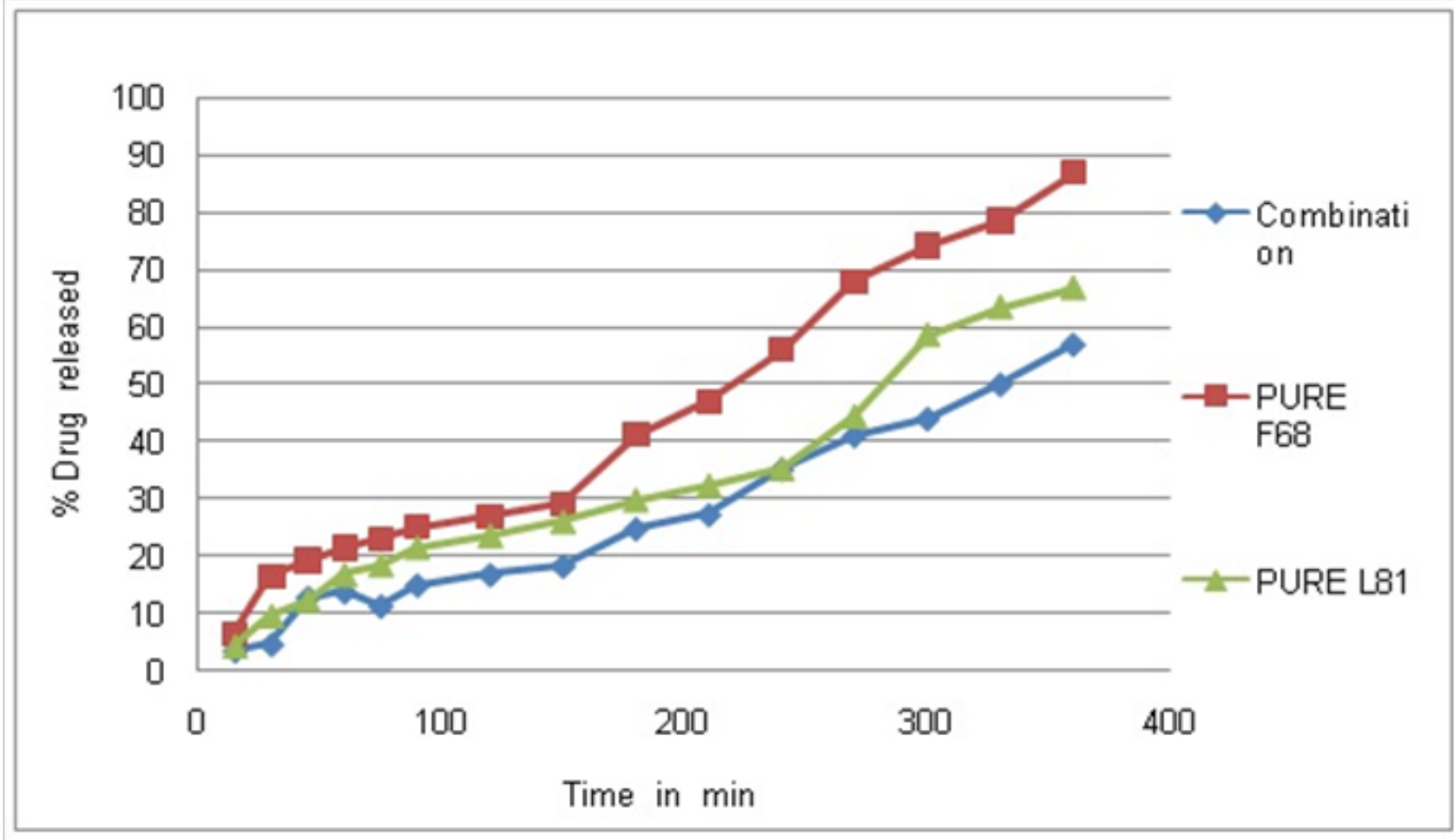

Figure 6: Comparison of drug release profiles of mono Pluronic F 68,Pluronic L81 micelles and their mixed micelles in pH 6 Buffer

behavior of Lamotrigine indicated the stability of Lamotrigine incorporation into the mixed micelles. The differences in the drug release rate reflect the advantages offered by the developed mixed micellar system over mono micellar system. A slow release even under the in vitro sink condition shows the Lamotrigine incorporated into the inner hydrophobic compartment stayed securely by the micelles.

Micelle storage stability
Lamotrigine-loaded plain and mixed micelles prepared from two different polymer types with the highest loading efficiency were used in stability study. Micellar structure is strongly depend on temperature. The temperature at which micelles are formed is known as critical micellization temperature. The micelles were lose their intact structure and drug would precipitate, if micelles were kept in refrigerator, temperature would fall lower than critical micellization temperature. ${ }^{24}$ For this rea- 
son, stability test was performed at $25^{\circ}$. In the aqueous form of Lamotrigine-loaded pure Pluronic F68 \& L81, $7.1 \%, 5.625 \%$ and for mixed micelles $3.2 \%$ decrease in Lamotrigine content was observed which indicate that stability of mixed micelles were more stable than that of plain micelles. This may be due to degradation of Pluronic micelle structure. Drug content of lyophilized form of mixed Pluronic micelles did not change showing high storage stability than that of aqueous form.

\section{CONCLUSION}

In this study plain and mixed Pluronic micelles were prepared.The high drug loading and entrapment efficiency developed by mixed micelles promise a high solubilization potential of the binary system was higher than mono system of PluronicF68 and PluronicL81 for hydrophobic drugs. Solubility of a anticonvulsant drug Lamotrigine in the mixed Pluronic micelle investigated under the influence of different stimuli; such as changes in $\mathrm{pH}$ or temperature and with varying salt/copolymer concentration. The solubility of the Lamotrigine could be significantly enhanced by increasing copolymer concentration,temperature or with lowering $\mathrm{pH}$. The solubilization of Pluronic $\AA$ micelle is not favored in the presence of $\mathrm{NaCl}$. The sustained release behavior of drug from the mixed micellar system than plain miceller system since expects its role in controlled drug delivery.

\section{REFERENCEST}

1. Langer R. Drug delivery and targeting. Nature. 1998; 392(6679): 5-10.

2. Lipinski CA, Lombardo F, Dominy BW, Feeney PJ. Experimental and computational approaches to estimate solubility and permeability in drug discovery and development settings. Adv Drug Deliv Rev. 2001; 46: 3-26 .

3. Fernandez AM, Van Derpoorten K, Dasnois L. N-Succinyl-(beta-alanyl-Lleucyl-L-alanyl-L-leucyl) doxorubicin: an extra cellularly tumor-activated prodrug devoid of intravenous acute toxicity. J Med Chem . 2001; 44: 3750-3.

4. Groneberg DA, Giersig M, Welte T. Nanoparticle-based diagnosis and therapy. Curr Drug targets. 2006; 7: 643-8.

5. Farokhzad OC, Langer R. Nanomedicine: developing smarter therapeutic and diagnostic modalities. Adv Drug Deliv Rev. 2006; 58(14): 1456-9.

6. Aliabadi HM, Lavasanifar A. Polymeric micelles for drug delivery. Expert Opin. Drug Deliv. 2006; 12(36): 139-62.

7. Gaucher G, Marchessault RH, Leroux JC. Polyester-based micelles and nanoparticles for the parenteral delivery of taxanes. J. Control. Release. 2010; 143(1): 2-12.

8. Nakasima K, Bahadur P. Aggregation of water-soluble block copolymers in aqueous solutions: recent trends. Adv. Colloid Interface Sci. 2006; 123: 75 .

9. Riess G, Hurtrez G, Bahadur P. Block Copolymers, Encyclopaedia of Polymer Science and Engineering, 2nd ed., Wiley, New York; 1985.

10. Sezgin Z, Yuksel N, Baykara T. Preparation and characterization of polymeric micelles for solubilization of poorly soluble anticancer drugs. Eur. J. Pharm. Biopharm. 2006; 64(3): 261.

11. Chiappetta DA, Sosnik A. Poly (ethylene oxide)-poly (propylene oxide) block copolymer micelles as drug delivery agents: improved hydro solubility, stability and bioavailability of drugs. Eur. J. Pharm. Biopharm. 2007; 66(3): 303.
12. Oh KT, Bronich TK, Kabanov AV. Micellar formulations for drug delivery based on mixtures of hydrophobic and hydrophilic Pluronic block copolymers. J. Control. Release. 2004; 94(2): 411-22.

13. Mansur CRE, Barboza SP, González G, Lucas EF. PLURONIC x TETRONIC polyols: study of their properties and performance in the destabilization of emulsions formed in the petroleum industry. J. Colloid Interface Sci. 2004; 271: 232-40.

14. Allen CD, Maysinger A. Eisenberg, Nano-engineering block copolymer aggregates for drug delivery, Coll. Surf. B Biointerf. 1999; 16: 1-35.

15. Seizures and Epilepsy: Hope Through Research. National Institute of Neurological disorders and stroke. 2010 July26. Availaible from: URL: http:// www.ninds.nih.gov/disorders/epilepsy/detailepilepsy. htm (Assessed on $30^{\text {th }}$ July 2010)

16. Rxlist. Availaible From: URL: www.Rxlist.com. (Assessed on 30th July 2010)

17. Gao ZG, Fain HD, Rapoport N. Controlled and targeted tumor chemotherapy by micellar-encapsulated drug and ultra sound. J. Controlled Release. 2005; 102(1): 203.

18. Sharma, Rakesh, Bahadur P. Effect of different additives on the cloud point of a polyethylene oxide-polypropylene oxide-polyethylene oxide block copolymer in aqueous solution. Journal of Surfactants and Detergents. July 2002; 5(3): 263-8.

19. Sumbul Fatma, Reuven Yakubov, Kamran Anwar M, Mahmood Hussain. Pluronic L81 enhances triacylglycerol accumulation in the cytosol and inhibits chylomicron secretion. Journal of Lipid Research. 2006; 47(11): 2422-32.

20. Alexandridis P, Hatton TA. Poly (ethylene oxide)-poly-(propylene oxide)poly (ethylene oxide) block copolymer surfactant in aqueous solution and at interfaces: thermodynamics, structure, dynamics and modeling. Colloids surface A: Physicochem. Eng. Aspects. 1996; 96(1): 1-46.

21. LAMOTRIGINE-Lamotrigine tablet, Roxane Laboratories, Inc. Available from: URL. http://dailymed.nlm.nih.gov/dailymed/archives/fdaDruglnfo. cfm?archiveid $=20548$

22. Ronald Soong, Mu-Ping Nieh, Eric Nicholson, John Katsaras, Peter M, Macdonald. Bicellar mixtures containing pluronic F68: morphology and lateral diffusion from combined SANS and PFG NMR studies. Langmuir. 2010; 26(4): 2630-8.

23. Kadam Y, Bharatiya B, Hassan PA, Verma G, Aswal VK, Bahadur P. Effect of an amphiphilic diol $\left(\right.$ Surfynol $^{\circledR}$ ) on the micellar characteristics of PEO-PPOPEO block copolymers in aqueous solutions. Colloids Surf. A: Physico chem. Eng. Aspects. 2010; 363(1): 110.

24. Kabanov AV, Alakov VY. Pluronic block copolymers in drug delivery: from micellar nano containers to biological response modifiers, Crit. Rev. Ther. Drug Carrier Syst. 2002; 19(1): 1-73. 